\title{
ROBUST H.263 VIDEO TRANSMISSION OVER SPECTRALLY SHAPED CHANNELS USING MULTICARRIER MODULATION
}

\author{
H. Zheng and K.J.Ray Liu \\ Department of Electrical Engineering and Institute for System Research \\ University of Maryland,College Park, MD 20742 \\ e-mail: haitaoz, kjrliu@eng.umd.edu
}

\begin{abstract}
This paper proposes an efficient and robust technique for transmission of entropy coded video over noisy channel, especially for spectrally shaped channels which are commonly used in ADSL environment. Combining multicarrier modulation and the Error Resilient Entropy Coding (EREC) technique together, we develop a new loading algorithm which achieves unequal error protection (UEP) by optimally allocating the available channel resources among the subchannels. Without adding any redundancy, the proposed system offers significant improvement over the EREC system. Simulation results on the $\mathrm{H.263}$ video coder over typical spectrally shaped channels which are typical in ADSL are provided.
\end{abstract}

\section{INTRODUCTION}

In recent year, multicarrier modulation (MCM)[1] has been widely used in ADSL and generated a tremendous amount of interests. A crucial aspect in the design of MCM system is the need to optimize the system transmission bandwidth and power through an optimal loading algorithm. By assigning high modulation rates to carriers with high signal-to-noise ratio (SNR) and low modulation rates to carriers with low SNR, or even no transmission on the carriers with very low SNR $[1,2,3]$, all the usable subcarriers perform with the same error rate. Typical channels in ADSL are spectrally shaped channel as shown in Figure 1.

Recently, MCM was introduced into multimedia communications [4,5] to provide unequal error protection(UE ${ }^{3}$ ) by allowing source components of different importance to be transmitted in different subchannels and efficiently allocating the available resource, power and bandwidth to the subchannels. A new loading algorithm for transmitting subband coded image over spectrally shaped channels is developed in [5]. The source data is divided into different layers associated with different perceptual irnportance and transmitted through different subchannels, shown in Figure 1. As a result, UEP is achieved by efficiently assigning the power and modulation rate under the total power and throughput constraints. Such algorithm achieves significant performance improvement compared to the existing loading algorithms[3]. However, these schemes require 
the images and videos to be coded with fixed length code. In practice, many compression standards, such as JPEG, MPEG, H.261 and H.263[6] involve entropy coding, thus produce variable length data blocks. The compressed data is highly sensitive to the propagation of channel errors, especially when the errors cause the decoder to lose synchronization and produce dramatical performance degradation. Possible solutions such as inserting extra synchronization codewords and error correction codes [7] introduce more redundancy and thus less compression efficiency.

EREC was adapted to image and video coding[8] to give increased resilience to random and burst errors while maintaining high compression. The basic idea is to reorganize the variable length blocks to fixed length slots such that each block starts at a known position. We observe that although EREC algorithm can remove the synchronization loss, it cannot prevent or reduce the distortion caused by the errors happening to the data inside the slots. Current EREC scheme is designed to transmit the bits of each slot with the same BER. However, for entropy coded video, code bits are of different importance and should receive UEP. In this paper, we propose to combine MCM and EREC, adopting the flexibility of error performance design offered by MCM together with the fixed length and synchronization-loss-free advantage of EREC, to construct a powerful and reliable video transmission system.

\section{SYSTEM}

\section{- MCM system for Fixed Length Data Blocks}

The MCM system can be modeled as several parallel, independent subchannels, which allows the power, modulation and even the channel encoder/decoder of each subchannel to be changed flexibly without affecting other subchannels. For transmission of layered data with unequal importance, this property shows more promising advantages since it can provide different error protections for different layers. The channel error effect is not avoided but rather reduced by assigning important layers with more channel protection at the cost of less important layer with less channel protection. In [5], we have proposed a loading algorithm which efficiently allocates the transmission power, bandwidth (modulation rate) among the subchannels to minimize the channel distortion. Assuming the orthogonality of source and channel, the overall distortion can be written as the sum of source distortion and channel distortion. For a fixed source coding scheme, the source distortion is determined. Therefore, the objective of the MCM resource allocation is to find the best power and modulation rate combinations $\left\{E_{m}, R_{m}\right\}_{m=1}^{N}$ for the data layers as to minimize the channel distortion. Mathematically, for $N$ data layers, this is represented as

$$
\begin{array}{cl}
\text { Min } \quad & D_{c}=\sum_{m=1}^{N} \operatorname{Pe}\left(R_{m}, \frac{E_{m}}{N_{m}}\right) W_{m}, \\
\text { subject to } & \sum_{i=m}^{N} E_{m} \leq E_{T}, \quad \sum_{i=m}^{N} R_{m}=R_{T},
\end{array}
$$


where $P e$ represents the bit erro: rate function, $W_{m}$ represents the average distortion caused by a single bit error at layer $m$, defined as the importance weighting factor of layer $m$ while $E_{T}$ and $R_{T}$ are the power and bandwidth constraint, respectively. For QAM modulation, $P e$ can be represented as a function of $R_{m}, E_{m}$, noise variance $N_{m}$ and aumber of subchannel $c_{m}, \operatorname{Pe}\left(R_{m}, E_{m}, N_{m}\right) \approx$

$4 Q\left(\sqrt{\frac{3 E_{m}}{c_{m} N_{m}\left(2^{R_{m}}-1\right)}}\right)$. For fixed $\left\{R_{m}\right\}_{m=1}^{N}$, the optimal power allocation can be solved easily by applying Lagrange multiplier. The optimal $\left\{E_{m}, R_{m}\right\}_{m=1}^{N}$ combination can be derived by applying the loading algorithm developed in [5] with minor increase of complexity. Further details can be obtained in [5].

For spectrally shaped channelis, usually the noise variance and transmission gain vary dramatically from subchannel to subchannel. Figure 2 illustrates the error performance comparison arnong different loading algorithms. The traditional loading algorithms $[2,3]$ obtain "average" channel, while the proposed new loading algorithm divides the subchannels into "good" channels and "bad" channels. As such, important data is transmitted through "good" channels to prevent huge error effect and improve overall performance. It is also observed that above optimization requires the knowledge of the length of source layers, and thus only fixed length coded image applications is considered[5].

- MCM System for Variable Length Data with Unequal Importance Incorporating the EREC scheme proposed in [8], the fixed length requirement for the loading algorithm can be removed, since for EREC coded video, the slot size is fixed and the importance or the weighting factor of the layers inside the slots can be classified. We propose to combine EREC scheme with the MCM system into a robust, efficient compression and transmission system. As shown in Figure 2, the essence of this approach is first block coding the source data as usual and assigning the variable length blocks to fixed length slots by applying the EREC scheme. The bits belongs to each slot are classified into several layered components of different importance. The slot size and the frame type should be considered for classification. Furthermore, error correction codes can be selected flexibly at each subchannel to further improve the performance. Here no need of extra bits indicating the location of each layer. This can also be viewed as a combined source and channel coding approach where the source coding and channel coding are considered together.

\section{ROBUST H.263 VIDEO TRANSMISSION}

H.263 has been widely used for: low bit rate video coding applications [6]. Due to the strong correlations among the video sequence, temporal and spatial predictions are commonly used in video coder for more compression efficiency. Unfortunately, this also creates high noise sensitivity problem. To achieve robust transmission, we incorporate the combined MCM and EREC scheme into H.263 stream.

For intra frames, H.263 transmits the six blocks belong to the same macroblock consecutively, resulting in unknown location of intra-DCs. However, intra-DCs are the most important data for the intra frame and should receive 
highest protection. Therefore, we propose to move the six intra-DCs belonging to the same macroblock to the beginning of the macroblock and each coded as 8-bit codeword. This aims to obtain more precise layer classification and weighting factor computation and thus ensure the correctness and efficiency of the protection.

For inter-frames, each macroblock begins with the coded macroblock indication (COD) bit, indicating whether or not the macroblock is coded. It should be noted that error occurring to this bit will not only corrupt the current macroblock, but also propagate huge errors to other blocks. Therefore, we assign the highest importance to the COD bit. Next to the COD bit, the MCBPC (Macroblock type \& block pattern) and MVD (motion vector data) are classified into the middle importance class. Inside each block layer, the inter-DCs and inter-ACs are together entropy coded to further reduce the bit rate. Although DC are more important than ACs, they both are classified into the lowest importance class. Due to unpredictable length property of entropy coding, the importance weighting factor associate with the layers require intensive computation which is not quite practical. Instead, approximations are used. All the schemes associated with UEP has the same problem due to the variable length coding, but the proposed scheme has the advantage of fixed layer locations.

\section{SIMULATIONS AND RESULTS}

The simulations are carried out on the combined system and EREC system based on 60 frames of the standard QCIF $(174 \times 144$ pels $)$ color sequences "Trevor" and "Miss America". The coding, motion estimation and prediction are as same as the H.263 except changing the intra-DC codewords to fixed eight bits. We use a typical spectrally shaped channel in ADSL environment and following parameters: 256 subchannels, 512 bits throughput per FFT symbol. The $P S N R$ is an average of $Y, C_{b}$ and $C_{r}$ component, defined as $P S N R=\frac{4 P S N R_{Y}+P S N R_{C_{b}}+P S N R_{C_{r}}}{6}$. An intra frame is inserted every 16 frames at $30 \mathrm{fps}$ frame rate. Figure 3 (a)(c) show the the performance comparison. As can be seen, the combined system achieves $2-5 \mathrm{~dB}$ gain over the EREC system. Figure 3(b)(d) sketch the system performances as the PSNR value averaged over 60 frames at different average subchannel power, where approximately $2 \mathrm{~dB}-4 \mathrm{~dB}$ gain is achieved by combined system. Although error correction codes and error concealment can be applied to further improve the performance, we choose not to employ them to make clear the performance of the proposed scheme.

\section{CONCLUSION}

We have proposed a combined multicarrier modulation and error resilient entropy coding scheme as a robust and efficient transmission technique for variable length coded video. Under noisy channels, not only synchronization-loss free, the proposed system achieves significant performance improvement by assigning better error performance to the important data bits at the cost of less error 
performance to the less important data bits. Employing a new loading algorithm, unequal error protection can be flexibly achieved by optimally allocating the resources among the subchannels. The complexity of the combined system mainly depends on the implementation of EREC scheme, while the complexity of loading algorithm can be ignored. The proposed combined system can be used for all types of data that consists of layers with different importance.

\section{References}

[1] J.A.C. Bingham, "Multicarrier modulation for data transmission: An idea whose time has come", IEEE comm. Mag., May 1990, pp. 5-14.

[2] P.S. Chow etc., "A practical discrete multition transceiver loading algorithm for data transmission over spectrally shaped channels",IEEE Trans. on Comms, 773775, Feb 1995.

[3] R.F.H. Fisher and J.B. Huber, "A new loading algorithm for discrete multition transmission",Proc. of GlobalCOM 96, pp 724-728.

[4] K.P. Ho and J.M. Kahn, "Image transmission over noisy channels using multicarrier modulation", Signal Processing: Image Comm.

[5] H. Zheng and K.J.R Liu, "A New Loading Algorithm for Subband Coded Image Transmitting over Noisy Channel using Multicarrier Modulation", to appear Asiloma 98.

[6] ITU-T DRAFT H.263, Video Coding for Low Bitrate Communication, May 1996.

[7] C.W. Yap etc. "Error Protection scheme for the transmission of H.263 coded video over mobile radio channels", VCIP 97.

[8] D.W. Redmill and N.G. Kingsbury, "The EREC: An Error-Resilient Technique for Coding Variable-Length Blocks of Data", IEEE Trans. on Image Processing, pp 565-574, April 1996.
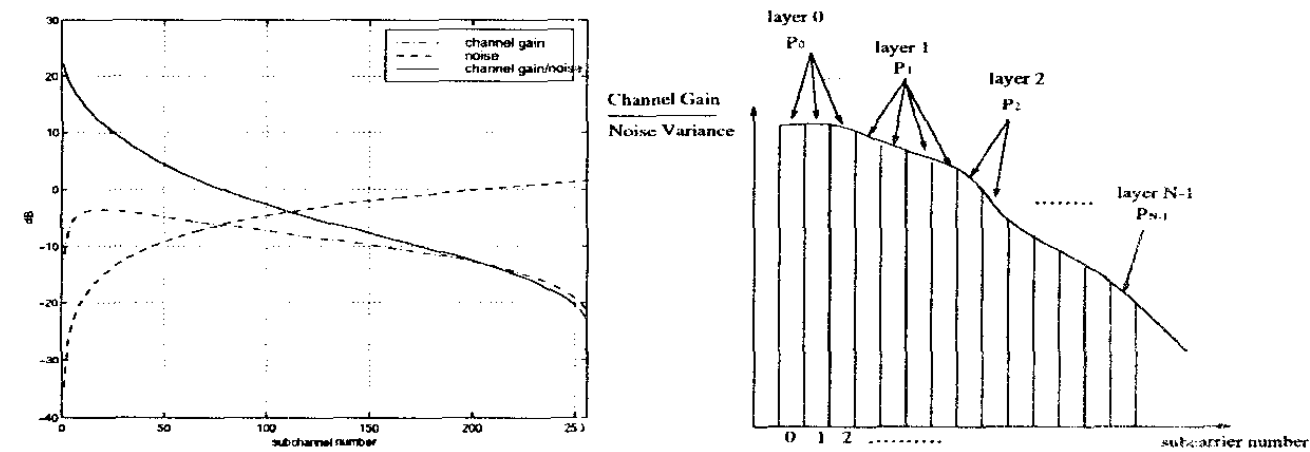

Figure 1: Left:Typical Spectrally, Shaped Channel;Right:Transmission model 

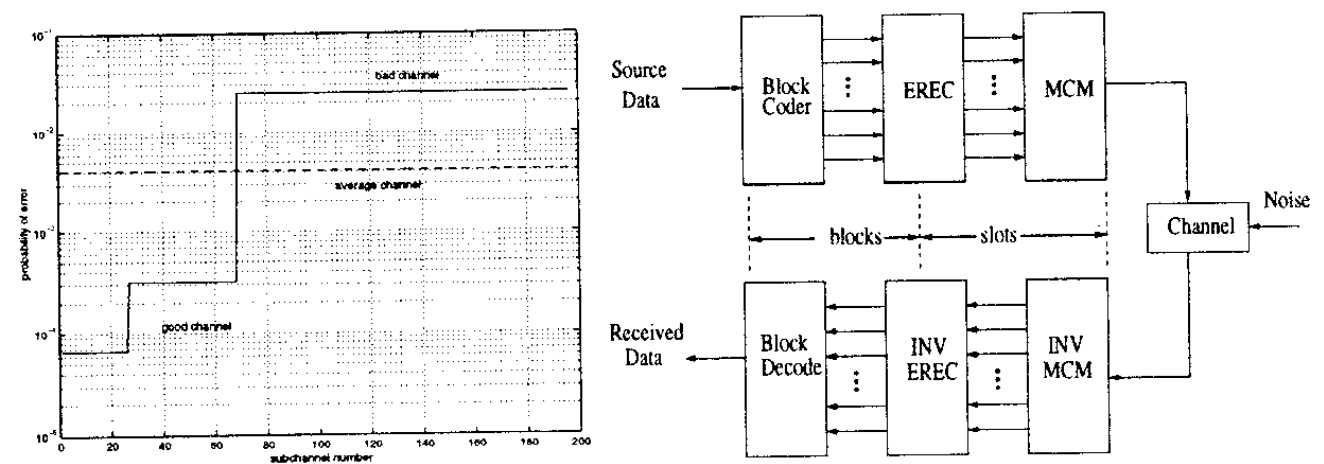

Figure 2: Left:subchannel BER ; Right: Block diagram of Combined MCM and EREC system

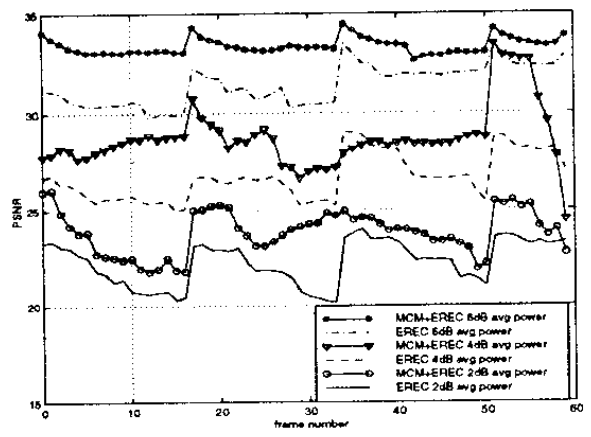

(a)

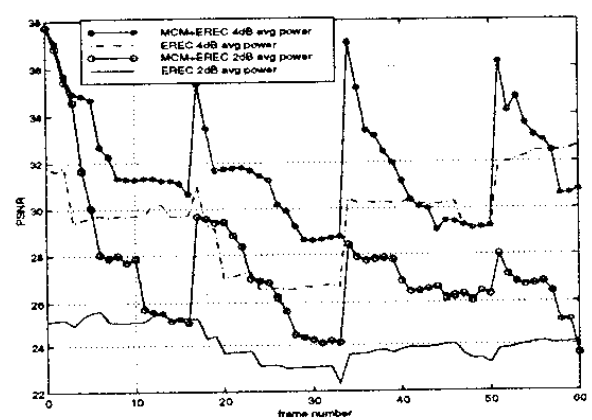

(c)

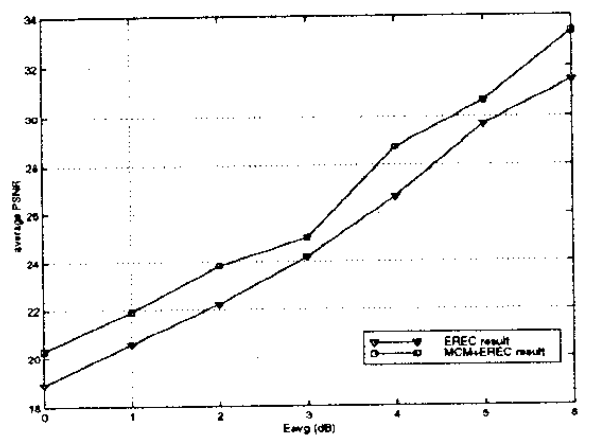

(b)

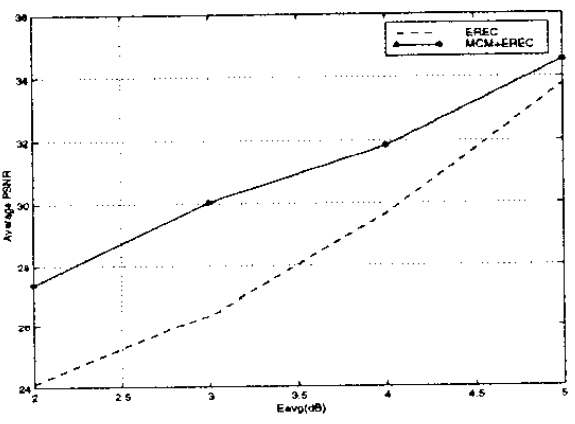

(d)

Figure 3: Performance comparison to [8]. "Trevor": (a) PSNR vs. frame number and (b) averaged PSNR; "Miss America": (c) PSNR vs. frame number and $(d)$ averaged PSNR. 